\title{
Directionality of EEG synchronization in Alzheimer's disease subjects
}

\author{
Claudio Babiloni ${ }^{\text {a,b,c,* }}$, Raffaele Ferri ${ }^{\mathrm{d}}$, Giuliano Binetti ${ }^{\mathrm{b}}$, Fabrizio Vecchio ${ }^{\text {a,c }}$, \\ Giovanni B. Frisoni ${ }^{b}$, Bartolo Lanuzza ${ }^{\mathrm{d}}$, Carlo Miniussi ${ }^{\mathrm{e}}, \mathrm{b}$, Flavio Nobili ${ }^{\mathrm{f}}$, \\ Guido Rodriguez $^{\mathrm{f}}$, Francesco Rundo ${ }^{\mathrm{d}}$, Andrea Cassarino ${ }^{\mathrm{a}}$, Francesco Infarinato ${ }^{\mathrm{a}}$, \\ Emanuele Cassetta ${ }^{\mathrm{c}}$, Serenella Salinari ${ }^{\mathrm{g}}$, Fabrizio Eusebi ${ }^{\mathrm{a}, \mathrm{h}}$, Paolo M. Rossini ${ }^{\mathrm{b}, \mathrm{c}, \mathrm{i}}$ \\ a Department of Human Physiology and Pharmacology, Univ. "La Sapienza" Rome, Italy \\ b IRCCS “S. Giovanni di Dio-F.B.F.", Brescia, Italy \\ ${ }^{\mathrm{c}}$ A.Fa.R. Osp. FBF, Isola Tiberina, Rome, Italy \\ ${ }^{\mathrm{d}}$ Department of Neurology, Oasi Institute for Research on Mental Retardation and Brain Aging (IRCCS), Troina, Italy \\ ${ }^{\mathrm{e}}$ Department of Biomedical Sciences and Biotechnologies, University of Brescia, Italy \\ ${ }^{\mathrm{f}}$ Division of Clinical Neurophysiology (DIMI), University of Genova, Italy \\ g Department of Informatics and Systems, Univ. "La Sapienza" Rome, Italy \\ ${ }^{\mathrm{h}}$ Institute of Sport Medicine and Science, CONI Services, Rome and Neuromed IRCCS, Isernia, Italy \\ i University "Campus Biomedico" Rome, Italy \\ Received 5 January 2007; received in revised form 27 April 2007; accepted 4 May 2007 \\ Available online 15 June 2007
}

\begin{abstract}
Is directionality of electroencephalographic (EEG) synchronization abnormal in amnesic mild cognitive impairment (MCI) and Alzheimer's disease (AD)? EEG data were recorded in 64 normal elderly (Nold), 69 amnesic MCI, and 73 mild AD subjects at rest condition (closed eyes). Direction of information flux within EEG functional coupling at electrode pairs was performed by directed transfer function (DTF) at delta $(2-4 \mathrm{~Hz})$, theta $(4-8 \mathrm{~Hz})$, alpha $1(8-10 \mathrm{~Hz})$, alpha $2(10-12 \mathrm{~Hz})$, beta $1(13-20 \mathrm{~Hz})$, beta $2(20-30 \mathrm{~Hz})$, and gamma $(30-40 \mathrm{~Hz})$. Parietal to frontal direction of the information flux within EEG functional coupling was stronger in Nold than in MCI and/or AD subjects, namely for alpha and beta rhythms. In contrast, the directional flow within inter-hemispheric EEG functional coupling did not discriminate among the three groups. These results suggest that directionality of parieto-to-frontal EEG synchronization is abnormal not only in AD but also in amnesic MCI. (C) 2007 Elsevier Inc. All rights reserved.
\end{abstract}

Keywords: Mild Alzheimer's disease (mild AD); Mild cognitive impairment (MCI); Electroencephalography (EEG); Directed transfer function (DTF)

\section{Introduction}

Amnesic mild cognitive impairment (MCI) is mainly characterized by a memory impairment that is insufficient to meet the criteria for the diagnosis of dementia (Flicker et al., 1991; Petersen et al., 1995, 2001; Portet et al., 2006). Annual conversion rate from normality to dementia of Alzheimer's type (Alzheimer's disease, AD) ranges between $0.2 \%$ and $4 \%$

\footnotetext{
* Corresponding author at: Dipartimento di Fisiologia Umana e Farmacologia, Università degli Studi di Roma "La Sapienza", P.le Aldo Moro 5, 00185 Rome, Italy. Tel.: +39 0649910989 ; fax: +39 0649910917.

E-mail address: claudio.babiloni@uniroma1.it (C. Babiloni). $U R L:$ http://hreeg.ifu.uniroma1.it/ (C. Babiloni).
}

(Petersen et al., 2001; Frisoni et al., 2004), whereas that from MCI to AD is between 6\% and 25\% (Petersen et al., 1999, 2001). It is an open issue with important clinical implications whether or not MCI is essentially a prodromic stage of $\mathrm{AD}$ (Petersen et al., 2001).

Despite encouraging previous evidence (Huang et al., 2000; Prichep et al., 2006), amplitude of electroencephalographic (EEG) rhythms alone might not predict individual conversion from MCI to dementia at the high statistical level required by clinical practice. A reasonable hypothesis is that the amplitude of EEG rhythms just partially captures one of the main features of $\mathrm{AD}$, namely the impairment of functional neural connectivity. In this vein, it has been reported that $A D$ patients present an abnormal linear coupling of EEG rhythms 
between cortical regions, as revealed by spectral EEG coherence (Jelic et al., 1997; Locatelli et al., 1998; Wada et al., 1998a, 1998b; Knott et al., 2000; Adler et al., 2003). Such a coherence denotes linear temporal synchronicity of coupled EEG rhythms as a reflection of coupled neural sources. It has been proposed that functional coupling of cortical rhythms is modulated by cholinergic systems (Xiang et al., 1998) and AD is characterized by a disruption of basal forebrain cholinergic inputs to cortex and hippocampus (Mesulam, 2004).

Most of the EEG studies in AD reported prominent decrease of EEG or magnetoencephalographic (MEG) alpha band coherence (Leuchter et al., 1992; Besthorn et al., 1994; Locatelli et al., 1998; Wada et al., 1998a, 1998b; Berendse et al., 2000; Jelic et al., 1997; Knott et al., 2000; Adler et al., 2003). Alpha coherence reduction in AD has been also found to be associated with an allelic pattern of ApoE genetics risk, which is supposed to be mediated by cholinergic deficit (Nolte et al., 2004). Delta and theta coherence provided less straightforward findings (Adler et al., 2003; Brunovsky et al., 2003; Dunkin et al., 1994; Leuchter et al., 1992; Locatelli et al., 1998; Calderon Gonzalez et al., 2004). To improve the evaluation of EEG functional coupling, EEG or MEG data have been analyzed with several procedures inspired by the theory of nonlinear dynamical systems such as "synchronization likelihood" (Stam et al., 2003; Stam and van Dijk, 2002; Babiloni et al., 2004a, 2006b; Pijnenburg et al., 2004), which significantly decreased at $10-12 \mathrm{~Hz}, 14-18 \mathrm{~Hz}$ and $18-22 \mathrm{~Hz}$ bands in AD patients, when compared to MCI and/or Nold subjects (Stam et al., 2003; Stam and van Dijk, 2002; Babiloni et al., 2004a, 2006a; Pijnenburg et al., 2004).

Both spectral coherence and synchronization likelihood have an important limitation: they do not reflect the direction of the information flux within the functional coupling of EEG/MEG rhythms at paired brain sites. One can overcome this limitation by the computation of the directed transfer function (DTF; Kaminski and Blinowska, 1991), modeling directional information flux within linear EEG functional coupling, (Korzeniewska et al., 1997; Kaminski et al., 1997; Mima et al., 2000; Sulimov, 1998). In precedence, a dominant parietal to frontal directional flux within EEG coupling during visuo-spatial information processing has been reported in healthy awakening subjects (Babiloni et al., 2004c, 2006b). Based on such an evidence, the present EEG study tested the hypothesis of a reduction of parietal-to-frontal directional information flux within EEG functional coupling in both MCI and mild AD subjects, in line with the idea of a common pathophysiological background linking these conditions.

\section{Methods}

\subsection{Subjects}

For this study, 73 AD patients, 69 amnesic MCI patients, and 64 Nold subjects were recruited. Local institutional ethics committees approved the study. Informed consent was obtained from all participants or their caregivers, according to the Declaration of Helsinki and standards established by the Authors' Institutional Review Boards.

Probable AD was diagnosed according to NINCDSADRDA (McKahn et al., 1984) and DSM IV criteria. All recruited $\mathrm{AD}$ patients underwent general medical, neurological and psychiatric assessments. Patients were also rated with a number of standardized diagnostic and severity instruments that included the Mini Mental State Examination (MMSE, Folstein et al., 1975), the Clinical Dementia Rating Scale (CDR, Hughes et al., 1982), the 15-item version of the Geriatric Depression Scale (GDS, Yesavage et al., 1982-1983), the Hachinski Ischemic Scale (HIS, Rosen et al., 1980), and the Instrumental Activities of Daily Living scale (IADL, Lawton and Brodie, 1969). Neuroimaging diagnostic procedures (CT or MRI) and complete laboratory analyses were carried out to exclude other causes of progressive or reversible dementias, in order to have a homogenous AD patient sample. Exclusion criteria included, in particular, evidence of (i) frontotemporal dementia, (ii) vascular dementia based on clinical and radiological grounds, (iii) extrapyramidal syndromes, (iv) reversible dementias, and (v) fluctuations in cognitive performance and visual hallucinations (suggestive of a possible Lewy body dementia). Inclusion and exclusion criteria for amnesic MCI diagnosis aimed at selecting elderly persons with objective cognitive deficits, especially in the memory domain, who did not meet the criteria for dementia or AD (Petersen et al., 2001; Portet et al., 2006). Inclusion criteria for amnesic MCI subjects were: (i) objective memory impairment on neuropsychological evaluation, as defined by performances $\geq 1.5$ standard deviation below the mean value of age and education-matched controls for a test battery including Busckhe-Fuld and memory Rey tests; (ii) normal instrumental activities of daily living as documented by history and evidence of independent living as assessed by a formal questionnaire (IADL, see above); and (iii) a clinical dementia rating score of 0.5. Exclusion criteria for amnesic MCI were: (i) MCI subjects without objective memory deficits; (ii) AD, as diagnosed by the procedures described above; (iii) evidence of concomitant dementia such as frontotemporal, vascular dementia, reversible dementias (including dementia of depression), fluctuations in cognitive performance, and/or features of mixed dementias; (iv) evidence of concomitant extrapyramidal symptoms; (v) clinical and indirect evidence of depression as revealed by GDS scores greater than 14; (vi) other psychiatric diseases, epilepsy, drug addiction, alcohol dependence, and use of psychoactive drugs or drugs interfering with brain cognitive functions including acetylcholinesterase inhibitors; and (vii) current or previous uncontrolled systemic diseases or traumatic brain injuries.

Nold subjects were recruited mainly among the patients' spouses. All Nold subjects underwent physical and neurological examinations as well as cognitive screening (including MMSE). Subjects affected by chronic systemic illnesses (i.e. diabetes mellitus or organ failure) were excluded, as were subjects receiving psychoactive drugs. Subjects with a his- 
Table 1

Mean values $( \pm$ S.E.) of the demographic and clinical characteristics of the investigated cohort (i.e. 73 AD, 69 amnesic MCI, and 64 Nold subjects)

\begin{tabular}{llll}
\hline & AD & Amnesic MCI & Nold \\
\hline Subject & 73 & 69 & 64 \\
MMSE & $20.9 \pm 0.5$ & $26.6 \pm 0.2$ & $28.6 \pm 0.2$ \\
Age & $74.1 \pm 1.0$ & $74.1 \pm 0.8$ & $73.7 \pm 0.9$ \\
Education & $9.8 \pm 0.6$ & $9.8 \pm 0.5$ & $9.7 \pm 0.6$ \\
IAF & $8.6 \pm 0.2$ & $9.2 \pm 0.2$ & $9.2 \pm 0.1$ \\
Female/male & $35 \mathrm{~F} / 39 \mathrm{M}$ & $38 \mathrm{~F} / 31 \mathrm{M}$ & $32 \mathrm{~F} / 32 \mathrm{M}$ \\
\hline
\end{tabular}

tory of present or previous neurological or psychiatric disease were also excluded. All Nold subjects had a GDS score lower than 14.

Table 1 reports the mean values and standard error (S.E.) of relevant personal and clinical parameters of $\mathrm{AD}$, amnesic MCI, and Nold subjects.

\subsection{EEG recordings}

EEG data were recorded in resting subjects (eyes-closed; 0.3-70 Hz bandpass) from Fp1, Fp2, F7, F3, Fz, F4, F8, T3, C3, Cz, C4, T4, T5, P3, Pz, P4, T6, O1, O2 during late morning. To monitor eye movements, the electrooculogram (0.3-70 Hz bandpass) was also collected. All data were digitized in continuous recording mode ( 5 min of EEG; up to $256 \mathrm{~Hz}$ sampling rate). State of vigilance was controlled by visual inspection of EEG traces during recording session and subjects' drowsiness was avoided by verbal warnings. At the time of EEG recording, no patient received medications that could influence EEG rhythms such as benzodiazepines.

The EEG epochs with ocular, muscular and other types of artifacts were preliminary identified by a computerized automatic procedure (Moretti et al., 2003). Two expert electroencephalographers (blind to the diagnosis) doublechecked and confirmed visually the automatic selections. Finally, the EEG epochs contaminated by artifacts were removed.

\subsection{EEG power density spectrum}

For the analysis of EEG power density spectrum, artifactfree EEG epochs were re-referenced to a common average and were analyzed by a standard Fast Fourier Transform (FFT) algorithm (Matlab 6.5, Mathworks Inc., Natrick, MA). Frequency resolution was of $1 \mathrm{~Hz}$ (no phase shift). EEG relative power density was obtained normalizing the EEG absolute power density at each frequency bin and electrode for the mean of the EEG absolute power density across all frequency bins and electrodes. EEG bands of interest were delta $(1-4 \mathrm{~Hz})$, theta $(4-8 \mathrm{~Hz})$, alpha $1(8-10 \mathrm{~Hz})$, alpha $2(10-12 \mathrm{~Hz})$, beta $1(13-20 \mathrm{~Hz})$, beta $2(20-30 \mathrm{~Hz})$, and gamma $(30-40 \mathrm{~Hz})$.

The choice of fixed EEG bands did not account for the individual alpha frequency (IAF) peak, defined as the frequency associated with the strongest EEG power at the extended alpha range (Prichep et al., 2006). However, this should not affect the results, since most of the subjects had IAF peaks within alpha 1 band $(8-10 \mathrm{~Hz})$. Specifically, this was true for $87.5 \%$ in Nold subjects, for $78.2 \%$ in MCI subjects, and for $71.2 \%$ in $\mathrm{AD}$ patients.

\subsection{Statistical analysis of EEG power density spectrum}

Statistical analysis of the EEG power density spectra across each group allowed the qualitative evaluation of EEG data used as an input for the DTF analysis. Kolmogorov-Smirnov test showed that the values of EEG spectral power density did not fit Gaussian distribution in most cases. Therefore, we used square-root transformation to obtain a normal distribution of the EEG spectral power density. This allowed the use of ANOVA for the statistical analysis. The ANOVA factors (levels) were group (AD, MCI, Nold; independent variable), band (delta, theta, alpha 1 , alpha 2 , beta 1 , beta 2 , gamma), and electrode (F3, Fz, F4, C3, Cz, C4, P3, Pz, P4). The Mauchly's test evaluated the sphericity assumption. Correction of the degrees of freedom was made with the Greenhouse-Geisser procedure. Age, gender, education, and IAF were used as covariates. The Duncan test was used for post hoc comparisons $(p<0.05)$.

\subsection{DTF analysis: "direction" of the functional connectivity estimated by the Mvar model}

Before computing the DTF, the EEG data (original electrode reference according to Kaminski and Blinowska, 1991) were preliminarily normalized by subtracting the mean value and dividing by the variance, according to standardized rules by Kaminski and Blinowska (1991). An important step of the DTF method was the computation of the so-called multivariate autoregressive (Mvar) model (Kaminski and Blinowska, 1991; Korzeniewska et al., 1997; Kaminski et al., 1997; Kus et al., 2004; Blinowska et al., 2004). EEG data at 19 electrodes were simultaneously given as an input to the Mvar model towards the computation of the directional information flux among all the pair combinations of these electrodes. This model was used to estimate the "direction" of the information flow within the EEG rhythms between the frontal and parietal regions (F3-P3, Fz-Pz, F4-P4) and the left and right hemispheres (F3-F4, C3-C4, P3-P4).

The mathematical core of the Mvar algorithm used in this work is based on the fitting multivariate autoregressive model (ARfit), running on the platform Matlab 6.5. The model order was 7, as estimated by the Akaike criterion suggested in previous DTF studies (Kaminski and Blinowska, 1991; Korzeniewska et al., 1997; Kaminski et al., 1997; De Gennaro et al., 2004, 2005; Bertini et al., 2006). The goodness of fit was evaluated by visual inspection of the values of noise matrix $\mathrm{V}$ of the Mvar model, as defined in previous studies (Mima et al., 2001; Babiloni et al., 2004a, 2004b, 2004c). 
The anterior-posterior directional flow of information of EEG functional coupling was indexed as "parietal-to-frontal" minus "frontal-to-parietal" DTF values, namely anteriorto-posterior DTFdiff values. Positive anterior-to-posterior DTFdiff values indicated a prevalence of parietal-to-frontal over frontal-to-parietal direction of the information flux. Similarly, inter-hemispheric directional flow of information of EEG functional coupling was indexed as "right-toleft" minus "left-to-right" direction of the DTF values; namely, inter-hemispheric DTFdiff values. Positive interhemispheric DTFdiff values indicated the predominance of "right-to-left" over "left-to-right" direction of the DTF values.

The fact that the DTF analysis was done on the difference between the two reciprocal DTF directions should require a careful interpretation of the results. A zero value of such a difference meant equivalence of the two opposite DTF directions within the period of EEG data acquisition; namely, that the DTF directions were equally strong or equally weak or both equal to zero in the EEG period taken into account. Of note, the DTF solutions do not depend on the effects of electrode reference and head volume conduction, when electrodes are distributed over the whole scalp (Kaminski and Blinowska, 1991; Korzeniewska et al., 1997; Kaminski et al., 1997).

\subsection{Statistical analysis of DTFdiff values}

Kolmogorov-Smirnov test showed that the DTFdiff values fitted Gaussian distribution, so that ANOVA was used for the statistical analysis. The Mauchley test evaluated the sphericity assumption and correction of the degrees of freedom was carried out using the Greenhouse-Geisser procedure. Age, gender, education, and IAF were used as covariates. The Duncan test was used for post hoc comparisons $(p<0.05)$.

The ANOVA of the anterior-to-posterior DTFdiff values included the factors group (AD, amnesic MCI and Nold; independent variable), band (delta, theta, alpha 1, alpha 2, beta 1, beta 2, gamma), and electrode pair (F3-P3, Fz-Pz, and F4-P4). In the ANOVA of the inter-hemispheric DTFdiff values, the factor electrode pair comprised the electrode pairs F3-F4, C3-C4 and P3-P4.

\section{Results}

\subsection{EEG power density spectrum}

Fig. 1 illustrates the grand average of the transformed EEG spectral power density values computed in the Nold,

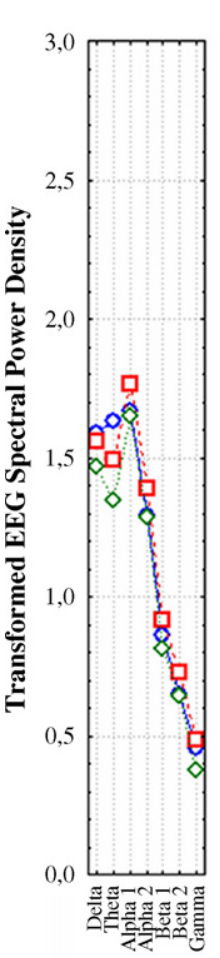

F3

$-\infty \mathrm{AD}$

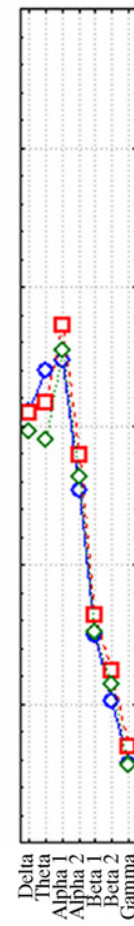

$\mathrm{Fz}$

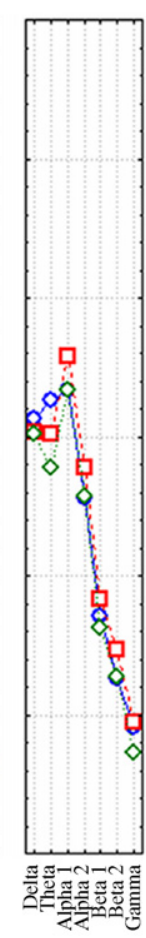

F4

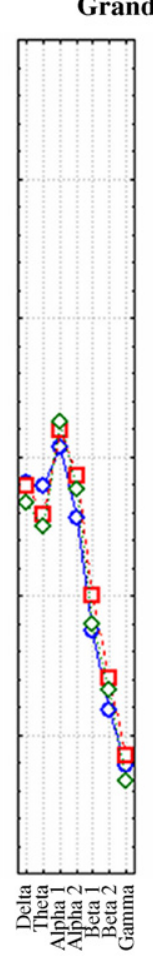

C3

$\cdots \diamond \cdots+$ Nol

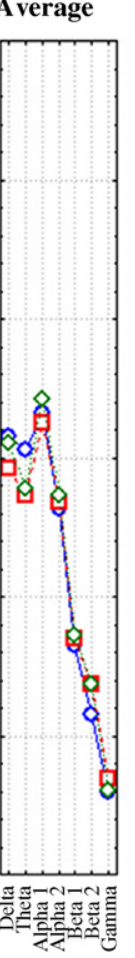

$\mathrm{Cz}$

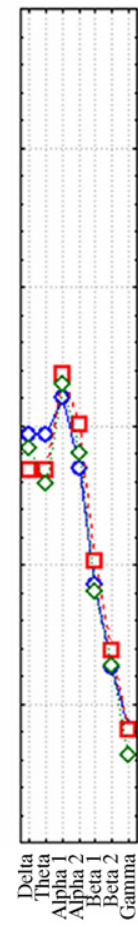

C4

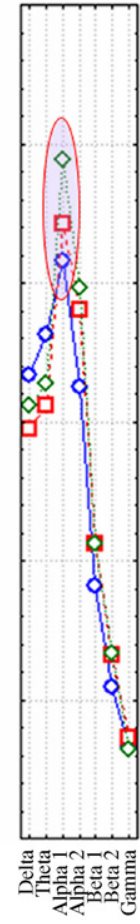

P3

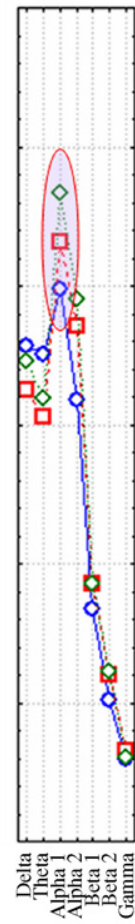

$\mathrm{Pz}$

Nold $>$ MCI $>$ AD $\mathrm{p}<\mathbf{0 . 0 2}$

Fig. 1. Grand average of the transformed EEG spectral power density values computed in the Nold, amnesic MCI, and AD subjects for the electrodes of interest (F3, Fz, F4, C3, Cz, C4, P3, Pz and P4) and for all frequency bands considered (delta, theta, alpha 1, alpha 2, beta 1, beta 2, gamma). 
amnesic MCI, and $\mathrm{AD}$ subjects for the electrodes of interest (F3, Fz, F4, C3, Cz, C4, P3, Pz and P4) and for all frequency bands considered (delta, theta, alpha 1, alpha 2, beta 1, beta 2 , gamma). Nold subjects showed maximum power density values at alpha 1 band in the posterior regions; minimum values of EEG power density were detected at high frequency bands (beta and gamma). Compared to Nold subjects, AD subjects were characterized by lower power density values at alpha 1 and by higher power density values at delta. The amnesic MCI subjects presented intermediate values of alpha 1 power density.

ANOVA of the transformed EEG spectral power density values showed a significant interaction $(F(96,9888)=2.08$; $p<0.00001)$ among the factors group (AD, amnesic MCI, Nold; independent variable), band (delta, theta, alpha 1, alpha 2, beta 1, beta 2, gamma), and electrode (F3, Fz, F4, C3, Cz, $\mathrm{C} 4, \mathrm{P} 3, \mathrm{Pz}, \mathrm{P} 4)$. Duncan post hoc testing indicated that the transformed EEG power density spectra matched the pattern Nold $>\mathrm{MCI}>\mathrm{AD}$ at alpha 1 band $(\mathrm{P} 3, \mathrm{P} 4, \mathrm{Pz} ; p<0.002$ to $p<0.0000001)$ and the pattern $\mathrm{AD}>\mathrm{MCI}$ at delta and theta bands (P3, P4, Pz; $p<0.01$ to $p<0.000001$ ). These results disclosed the expected "slowing" of resting EEG rhythms in the $\mathrm{AD}$ and MCI patients.

ANOVA of the transformed EEG spectral power density values showed a significant interaction $(F(96,9888)=2.08$; $p<0.00001)$ among the factors group (AD, amnesic MCI, Nold; independent variable), band (delta, theta, alpha 1, alpha
2, beta 1, beta 2, gamma), and electrode (F3, Fz, F4, C3, Cz, $\mathrm{C} 4, \mathrm{P} 3, \mathrm{Pz}, \mathrm{P} 4)$. Duncan post hoc testing indicated that the transformed EEG power density spectra matched the pattern Nold $>$ MCI $>$ AD at alpha 1 band (P3, P4, Pz; $p<0.002$ to $p<0.0000001)$ and the pattern $\mathrm{AD}>\mathrm{MCI}$ at delta and theta bands (P3, P4, Pz; $p<0.01$ to $p<0.000001)$. These results disclosed the expected "slowing" of resting EEG rhythms in the $\mathrm{AD}$ and $\mathrm{MCI}$ patients.

\section{2. $D T F$}

The Nold subjects showed wide positive anteriorposterior DTFdiff values (parietal-to-frontal DTF values prevailing over frontal-to-parietal values), which were maximum in magnitude at alpha 1 for all electrode pairs of interest (F3-P3, Fz-Pz, F4-P4). Compared to Nold subjects, AD patients were characterized by a decrease of these DTFdiff values. MCI subjects presented a DTF trend similar to that of the $\mathrm{AD}$, except for alpha 1 , alpha 2 , and beta 1 in which they showed intermediate values of anterior-to-posterior DTFdiff values, when compared to those of Nold and AD. In contrast to the anterior-to-posterior, inter-hemispheric DTFdiff values had similar magnitude values in the three groups, for all electrode pairs of interest (F3-F4, C3-C4, P3-P4).

Statistical ANOVA analysis of the anterior-to-posterior DTFdiff values showed a two-way ANOVA interaction $(F(12,1218)=3.49 ; p<0.00001)$ between the factors group

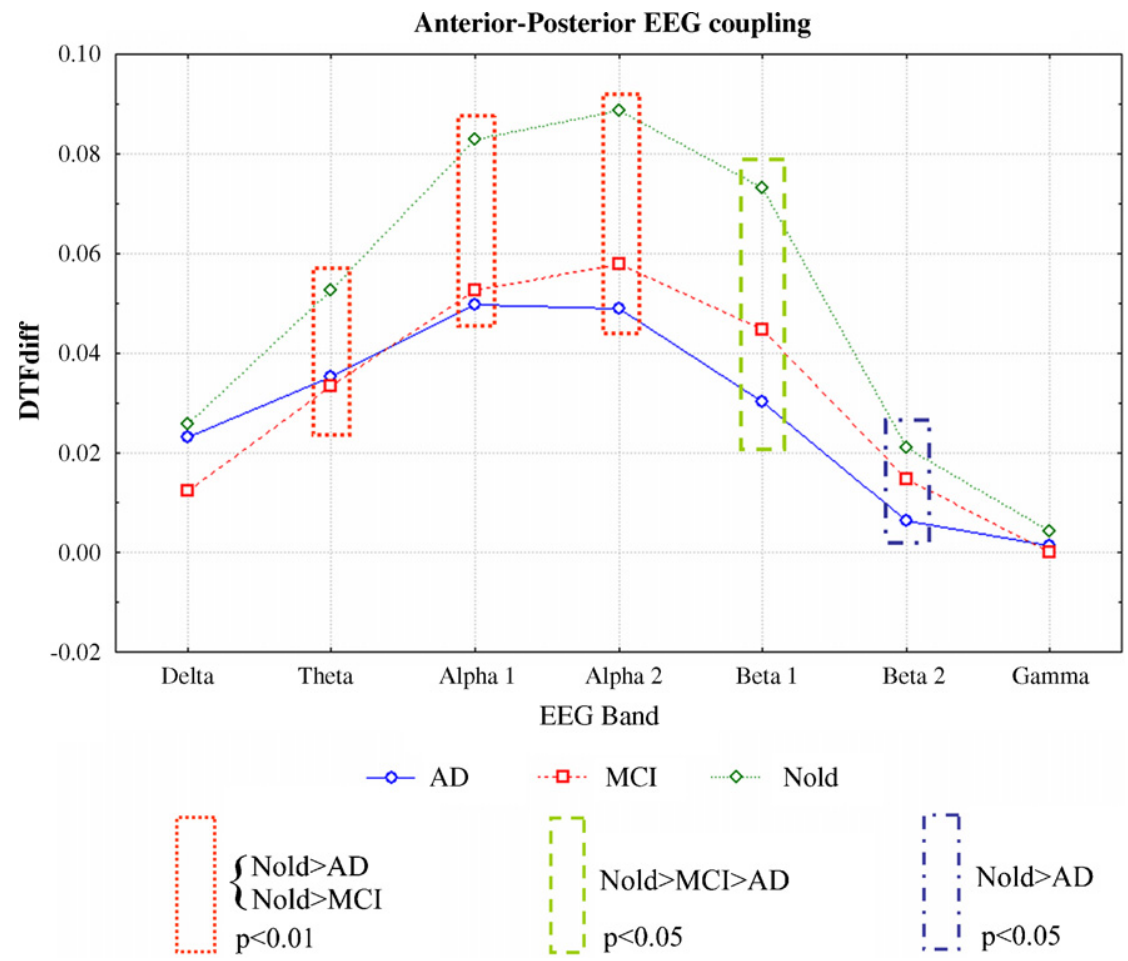

Fig. 2. Means of anterior-posterior DTFdiff values computed in the Nold, amnesic MCI, and AD for all frequency bands of interest (delta, theta, alpha 1, alpha 2, beta 1, beta 2, gamma). These means were obtained averaging the anterior-posterior DTFdiff values of the three electrode pairs (F3-P3, Fz-Pz, F4-P4), in order to represent a two-way ANOVA interaction $(F(12,1218)=3.49 ; p<0.00001)$ between the factors group $(\mathrm{AD}$, amnesic MCI, Nold) and Frequency band (delta, theta, alpha 1 , alpha 2 , beta 1 , beta 2 , gamma). 


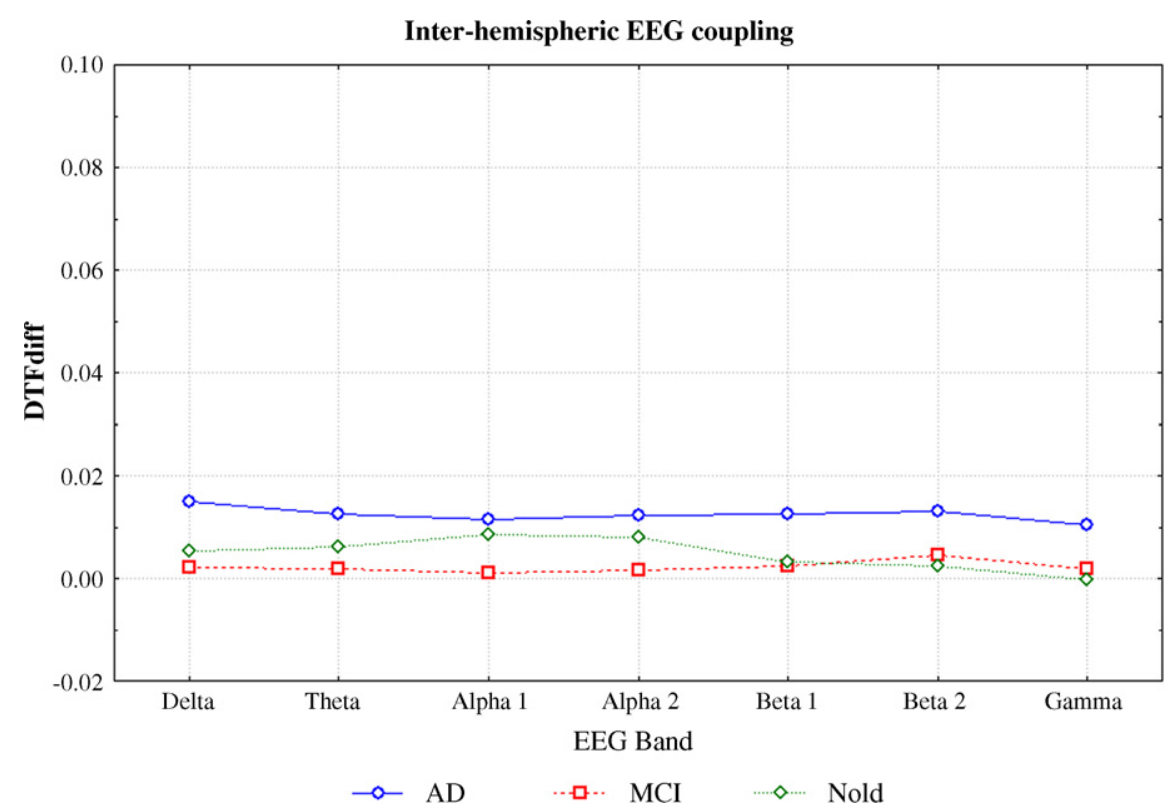

Fig. 3. Means of inter-hemispheric DTFdiff values computed in the Nold, amnesic MCI, and AD subjects at all frequency bands of interest (delta, theta, alpha 1, alpha 2, beta 1, beta 2, gamma). Means were obtained averaging the inter-hemispheric DTFdiff values of the electrode pairs (F3-F4, C3-C4, P3-P4), to provide data to be compared to those illustrated.

(AD, amnesic MCI, Nold) and Frequency band (delta, theta, alpha 1, alpha 2, beta 1, beta 2, gamma). Duncan post hoc testing showed that the anterior-posterior DTFdiff values matched the patterns Nold $>\mathrm{MCI}>\mathrm{AD}$ (beta 1 : $p<0.05$ to $p<0.000001$ ), Nold $>\mathrm{AD}$ (theta: $p<0.01$; alpha 1: $p<0.000005$; alpha 2: $p<0.000005$; beta $2: p<0.05)$, and Nold $>$ MCI (theta: $p<0.01$; alpha 1: $p<0.00001$; alpha 2: $p<0.00001)$. Fig. 2 shows the mean anterior-to-posterior DTFdiff values computed in the Nold, amnesic MCI, and AD subjects, for all frequency bands of interest (delta, theta, alpha 1 , alpha 2 , beta 1 , beta 2 , gamma), obtained by averaging the anterior-to-posterior DTFdiff values of the three electrode pairs (F3-P3, Fz-Pz, F4-P4). These values represent the above-mentioned two-way ANOVA interaction.

Statistical ANOVA analysis of the inter-hemispheric DTFdiff values showed no statistically significant effect including the factor group (AD, amnesic MCI, Nold). Fig. 3 reports the mean inter-hemispheric DTFdiff values computed in the Nold, amnesic MCI, and AD subjects at all frequency bands of interest (delta, theta, alpha 1, alpha 2, beta 1, beta 2 , gamma), obtained by averaging the inter-hemispheric DTFdiff values of the three electrode pairs (F3-F4, C3-C4, P3-P4).

\section{Control analysis}

It may be argued that the above DTF results were affected by different signal-to-noise ratio of resting EEG data in frontal as compared to parietal regions (see Nolte et al., 2004). To address this issue, EEG data were mathematically generated to fit uncorrelated noise (256 frequency sampling; 60 segments of EEG signals lasting $2 \mathrm{~s}$ each; 19 virtual "electrodes" named according to 10-20 system notation). Of note, we induced EEG power density ten times higher at the 3 parietal "electrodes" P3, Pz, P4 then at the remaining 16 "electrodes" (Fig. 4A). In line with the main DTF study, the DTF was computed taking into account the simulated EEG data of all 19 virtual "electrodes". Furthermore, the directional information flow of the simulated EEG data was indexed subtracting "parietal-to-frontal" to "frontal-to-parietal" DTF values (F3-P3, Fz-Pz, F4-P4), in order to obtain anterior-posterior DTFdiff values. As mentioned above (see Section 2), positive DTFdiff values point to a prevalence of parietal-to-frontal over frontal-to-parietal direction of EEG functional coupling. The results of this control analysis showed DTFdiff values very close to zero, thus indicating no preponderant parietal-to-frontal directionality (Fig. 4B). This was true despite the high power density of the simulated EEG data at the parietal virtual "electrodes" (P3, $\mathrm{Pz}, \mathrm{P} 4)$. It was concluded that DTF values are insensitive to uncorrelated noise with different power density.

\section{Discussion}

In the present study, we tested the hypothesis that directional flow within EEG functional coupling leads support to the idea that amnesic MCI is a pre-clinical condition for $\mathrm{AD}$ at group level. Results showed that the parietal-to-frontal EEG functional coupling was stronger in Nold than in amnesic MCI and/or AD subjects, at theta, alpha, and beta rhythms. In contrast, inter-hemispheric EEG information flux did not discriminate among the three groups. These results extend previous evidence of EEG synchronization likelihood show- 
(A)

\section{Spectral Power Density of the simulated EEG Data}
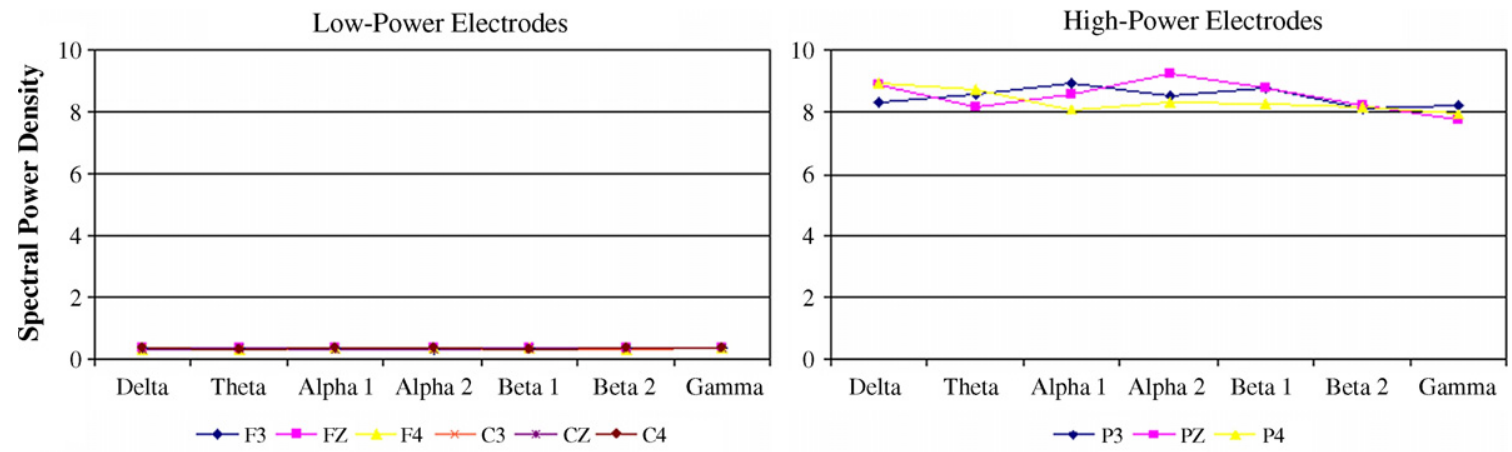

(B)

Antero-Posterior DTFdiff of the simulated EEG Data

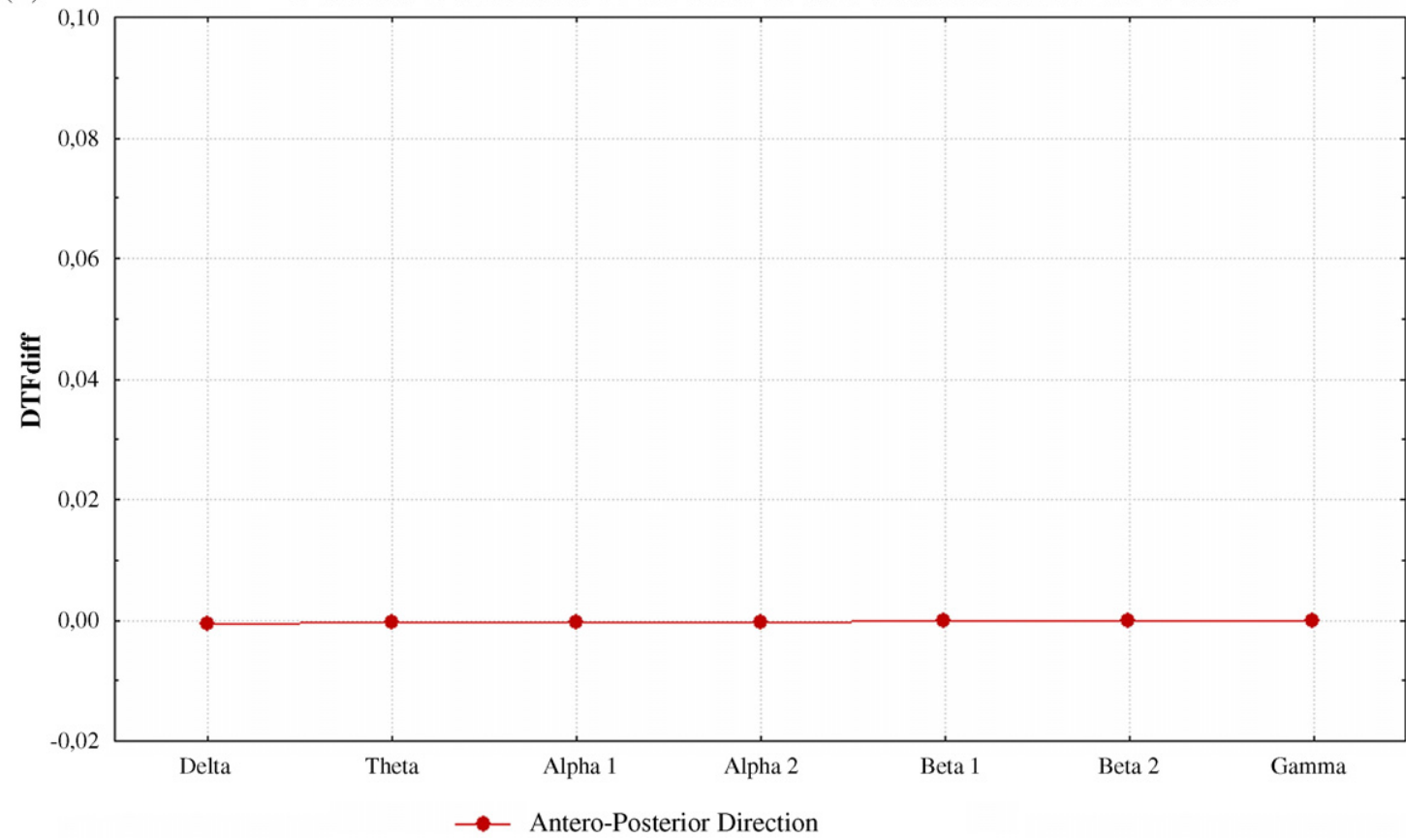

Fig. 4. (A) Spectral power density of mathematically generated EEG signals ( $2 \mathrm{~min} ; 256 \mathrm{~Hz}$ sampling frequency) at nine virtual "electrodes" of interest F3, Fz, F4, C3, Cz, C4, P3, Pz, P4 for delta, theta, alpha 1, alpha 2, beta 1, beta 2, and gamma bands. The simulated EEG data fitted uncorrelated noise. Of note, EEG power density was ten times higher at three parietal "electrodes" (P3, Pz, P4) then at the remaining "electrodes". (B) Mean of the frontal-parietal DTFdiff values computed from the simulated EEG data at F3-P3, Fz-Pz, and F4-P4 for all frequency bands of interest (delta, theta, alpha 1, alpha 2, beta 1, beta 2, and gamma). These values are very close to zero (no preponderant direction) and can be directly compared to the corresponding DTFdiff values of Fig. 3.

ing that fronto-parietal rather than inter-hemispheric EEG functional coupling is impaired in mild $\mathrm{AD}$ when compared to Nold, amnesic MCI, and vascular dementia (Babiloni et al., 2004a, 2006a). Furthermore, the present results agree with previous findings showing that global functional coupling of alpha rhythms is lower in $\mathrm{AD}$ patients with respect to amnesic MCI and/or Nold subjects (Stam et al., 2003; Pijnenburg et al., 2004). Finally, they complement previous MEG findings disclosing poor alpha functional coupling in AD (Stam et al., 2003).

The results of the present study raise at least two questions: "What is the physiological meaning of the parietal-to-frontal directional flux within the alpha and beta functional coupling?" and "What is the heuristic value of the above results in the study of pathological aging?" Delta (about $1-4 \mathrm{~Hz})$ and theta $(4-8 \mathrm{~Hz})$ characterize slow-wave sleep and mainly reflect thalamo-cortical interactions (Steriade, 2003). In awakening individuals, theta rhythms characterize hippocampal/parahippocampal-cortical interactions for focused attention, working memory, and encoding processes (Klimesch, 1999). Furthermore, alpha (about 8-12 Hz) and beta $(>12 \mathrm{~Hz})$ would be mainly related to attention and access to semantic memory (Rossini et al., 1991; Steriade and Llinas, 1988; Klimesch, 1996; Klimesch et al., 1997, 1998), reflecting the engagement of forebrain cholinergic pathways, thalamo-cortical, and cortico-cortical connectivity (Pfurtscheller and Lopez da Silva, 1999; Ricceri et al., 2004). Keeping these considerations in mind, parietal-to-frontal 
directional flow within theta, alpha, and beta functional coupling might reflect an effective integration among representations of external stimuli and cognitive representations within frontal-to-parietal and hippocampal/parahippocampal networks. Such connectivity might be defective in both amnesic MCI and AD conditions, in which there would be not only the loss of long distance axons but also the impairment of (cholinergic) basal forebrain projections to cortex and hippocampus (Kleiner and Bringmann, 1996; Mesulam, 2004). This explanation is in accordance with the important role of the cholinergic systems in attention (Cheal, 1981; Broks et al., 1988; Callahan et al., 1993) and with the disruptive effects of cholinergic antagonists (e.g. scopolamine) on EEG functional coupling (Breakspear, 2002; Friston, 2000a, 2000b, 2000c; Rodriguez et al., 1999).

The present results extend previous DTF evidence (Kaminski and Blinowska, 1991; Korzeniewska et al., 1997; Kaminski et al., 1997; Mima et al., 2000; Babiloni et al., 2004c, 2006b) showing that intrinsic parietal-to-frontal functional connectivity would characterize the awake rest and pre-sleep period (Huang et al., 2000; Hughes et al., 1982). It can be speculated that the preponderant parietalto-frontal over frontal-to-parietal EEG functional coupling would reflect a back-ground flux of sensory signals from parietal to frontal areas. This parietal-to-frontal flux might be actively regulated by a re-entrant (possibly inhibitory) frontal-to-parietal flux of signals. The strength of that reentrant flux would increase in the case of enhanced cognitive demands (Babiloni et al., 2004c, 2006b) or in the case of abnormal functional connectivity associated with $\mathrm{AD}$ process. Furthermore, the present results indicate that interhemispheric directional flux within EEG functional coupling is much less able than the frontal-to-parietal one to differentiate amnesic MCI from normal and mild AD subjects, in accordance with the functional effects of corpus callosum atrophy (Montplaisir et al., 1990; Yamauchi et al., 1993; Pinkofsky et al., 1997; Teipel et al., 1999; Knyazeva and Innocenti, 2001; Pogarell et al., 2005) and with the reduction of the inter-hemispheric functional coupling at slow EEG rhythms in physiological and pathological aging (Wada et al., 1998b; Kikuchi et al., 2000; Adler et al., 2003). That speculation is in line with the idea that integration of information processed in cerebral cortex might depend on the formation and dissolution of synchronized neuron assemblies characterized by various frequency bands and different directions of information flow (Engel and Singer, 2001; Singer, 2001; Sarnthein et al., 1998).

\section{Conclusions}

It has been shown previously that frontal-to-parietal direction of information flux within EEG functional coupling is an intrinsic feature of cerebral connectivity (Babiloni et al., 2004a, 2004c, 2006a, 2006b). Here we tested whether that direction of information flux is abnormal in pathological aging conditions such as amnesic $\mathrm{MCI}$ and $\mathrm{AD}$, in line with the hypothesis that amnesic MCI is a pre-clinical stage of $\mathrm{AD}$ at the group level. Results showed that parietal-to-frontal direction of the information flux within EEG functional coupling was stronger in Nold than in amnesic MCI and/or AD subjects, principally at alpha and beta rhythms. In contrast, the directional flow within inter-hemispheric EEG functional coupling did not discriminate among the three groups. These results suggest that directional information flux within EEG frontal-to-parietal coupling is quite sensitive to the preclinical stage of the AD at the group level. Future studies should evaluate the extent to which that abnormal linear "directionality" of cortical functional coupling, as suggested by DTF results, reflects changes of underlying thalamic rhythmic generators. They should also evaluate non-linear components of such a "directionality" (Jeong, 2004; Nolte et al., 2004; Pereda et al., 2005).

\section{Disclosure statement}

There are no conflicts on interest, or financial disclosures. The manuscript is not being considered for publication by any other journal. The corresponding author takes full responsibility of the integrity of the entire data. All authors have seen and agree to the final submitted version.

\section{Acknowledgments}

We thank Dr. Claudio Del Percio, Mrs. Rita Fini, Dr. Massimo Gennarelli, Dr. Nicola Girtler, and Dr. Franca Tecchio for their precious help in the development of the present study. We thank also Prof. Fabrizio Eusebi for his continuous support. Dr. Andrea Cassarino participated to this study in the framework of his Ph.D. program at the Doctoral School in Neurophysiology, University of Rome "La Sapienza".

\section{References}

Adler, G., Brassen, S., Jajcevic, A., 2003. EEG coherence in Alzheimer's dementia. J. Neural. Transm. 110 (9), 1051-1058.

Babiloni, C., Ferri, R., Moretti, D.V., Strambi, A., Binetti, G., Dal Forno, G., Ferreri, F., Lanuzza, B., Bonato, C., Nobili, F., Rodriguez, G., Salinari, S., Passero, S., Rocchi, R., Stam, C.J., Rossini, P.M., 2004a. Abnormal fronto-parietal coupling of brain rhythms in mild Alzheimer's disease: a multicentric EEG study. Eur. J. Neurosci. 19 (9), 25832590.

Babiloni, C., Binetti, G., Cassetta, E., Cerboneschi, D., Dal Forno, G., Del Percio, C., Ferreri, F., Ferri, R., Lanuzza, B., Miniussi, C., Moretti, D.V., Nobili F., Pascual-Marqui, R.D., Rodriguez, G., Romani, G.L., Salinari, S., Tecchio, F., Vitali, P., Zanetti, O., Zappasodi, F., Rossini, P.M., 2004b. Mapping distributed sources of cortical rhythms in mild Alzheimer's disease. A multi-centric EEG study. NeuroImage 22 (1), 57-67.

Babiloni, C., Babiloni, F., Carducci, F., Cincotti, F., Vecchio, F., Cola, B., Rossi, S., Miniussi, C., Rossini, P.M., 2004c. Functional frontoparietal connectivity during short-term memory as revealed by high-resolution EEG coherence analysis. Behav. Neurosci. 118 (4), 687-697. 
Babiloni, C., Ferri, R., Binetti, G., Cassarino, A., Forno, G.D., Ercolani, M., Ferreri, F., Frisoni, G.B., Lanuzza, B., Miniussi, C., Nobili, F., Rodriguez, G., Rundo, F., Stam, C.J., Musha, T., Vecchio, F., Rossini, P.M., 2006 a. Fronto-parietal coupling of brain rhythms in mild cognitive impairment: a multicentric EEG study. Brain Res. Bull. 69 (1), 63-73.

Babiloni, C., Vecchio, F., Cappa, S., Pasqualetti, P., Rossi, S., Miniussi, C., Rossini, P.M., 2006b. Functional frontoparietal connectivity during encoding and retrieval processes follows HERA model A high-resolution study. Brain Res. Bull. 68 (4), 203-212.

Berendse, H.W., Verbunt, J.P.A., Scheltens, Ph., van Dijk, B.W., Jonkman, E.J., 2000. Magnetoencephalographic analysis of cortical activity in Alzheimer's disease. A pilot study. Clin. Neurophysiol. 111, 604-612.

Bertini, M., Ferrara, M., De Gennaro, L., Curcio, G., Moroni, F., Vecchio, F., De Gasperis, M., Rossini, P.M., Babiloni, C., 2006. Directional information flows between brain hemispheres during pre-sleep wake and early sleep stages. Cereb. Cortex [Epub ahead of print].

Besthorn, C., Forstl, H., Geiger-Kabisch, C., Sattel, H., Gasser, T., SchreiterGasser, U., 1994. EEG coherence in Alzheimer disease. Electroenceph. Clin. Neurophysiol. 90, 242-245.

Blinowska, K.J., Kus, R., Kaminski, M., 2004. Granger causality and information flow in multivariate processes. Phys. Rev. E: Stat. Nonlin. Soft Matter Phys. 70 (5 Pt 1), 050902.

Breakspear, M., 2002. Nonlinear phase desynchronization in human electroencephalographic data. Hum. Brain Mapp. 15, 175-198.

Broks, P., Preston, G.C., Traub, M., Poppleton, P., Ward, C., Stahl, S.M., 1988. Modelling dementia: effects of scopolamine on memory and attention. Neuropsychologia 26, 685-700.

Brunovsky, M., Matousek, M., Edman, A., Cervena, K., Krajca, V., 2003. Objective assessment of the degree of dementia by means of EEG. Neuropsychobiology 48 (1), 19-26.

Calderon Gonzalez, P.L., Parra Rodriguez, M.A., Llibre Rodriguez, J.J., Gutierrez, J.V., 2004. Spectral analysis of EEG coherence in Alzheimer's disease. Rev. Neurol. 38 (5), 422-427.

Callahan, M.J., Kinsora, J.J., Harbaugh, R.E., Reeder, T.M., Davis, R.E., 1993. Continuous ICV infusion of scopolamine impairs sustained attention of rhesus monkeys. Neurobiol. Aging 14, 147-151.

Cheal, M.L., 1981. Scopolamine disrupts maintenance of attention rather than memory processes. Behav. Neural. Biol. 33, 163-187.

De Gennaro, L., Vecchio, F., Ferrara, M., Curcio, G., Rossini, P.M., Babiloni, C., 2004. Changes in fronto-posterior functional coupling at sleep onset in humans. J. Sleep Res. 13 (3), 209-217.

De Gennaro, L., Vecchio, F., Ferrara, M., Curcio, G., Rossini, P.M., Babiloni, C., 2005. Antero-posterior functional coupling at sleep onset: changes as a function of increased sleep pressure. Brain Res. Bull. 65 (2), 133-140.

Dunkin, J.J., Leuchter, A.F., Newton, T.F., Cook, I.A., 1994. Reduced EEG coherence in dementia: state or trait marker? Biol. Psychiatry 35, 870-879.

Engel, A.K., Singer, W., 2001. Temporal binding and the neural correlates of sensory awareness. Trends Cognit. Sci. 5, 16-25.

Flicker, C., Ferris, S.H., Reisberg, B., 1991. Mild cognitive impairment in the elderly: predictors of dementia. Neurology 41 (7), 1006-1009.

Folstein, M.F., Folstein, S.E., McHugh, P.R., 1975. 'Mini mental state': a practical method for grading the cognitive state of patients for clinician. J. Psychiat. Res. 12, 189-198.

Frisoni, G.B., Padovani, A., Wahlund, L.O., 2004. The predementia diagnosis of Alzheimer disease. Alzheimer Dis. Assoc. Disord. 18 (2), 51-53.

Friston, K.J., 2000a. The labile brain. I. Neuronal transients and nonlinear coupling. Philos. Trans. R. Soc. Lond. B: Biol. Sci. 355 (1394), 215-236, Review.

Friston, K.J., 2000b. The labile brain. II. Transients, complexity and selection. Philos. Trans. R. Soc. Lond. B: Biol. Sci. 355 (1394), 237-252, Review.

Friston, K.J., 2000c. The labile brain. III. Transients and spatio-temporal receptive fields. Philos. Trans. R. Soc. Lond. B: Biol. Sci. 355 (1394), 253-265, Review.

Huang, C., Wahlund, L., Dierks, T., Julin, P., Winblad, B., Jelic, V., 2000. Discrimination of Alzheimer's disease and mild cognitive impairment by equivalent EEG sources: a cross-sectional and longitudinal study. Clin. Neurophysiol. 111 (11), 1961-1967.

Hughes, C.P., Berg, L., Danziger, W.L., Cohen, L.A., Martin, R.L., 1982. A new clinical rating scale for the staging of dementia. Br. J. Psychiatry $140,1225-1230$.

Jelic, V., Julin, P., Shigeta, M., Nordberg, A., Lannfelt, L., Winblad, B., Wahlund, L.O., 1997. Apolipoprotein E epsilon4 allele decreases functional connectivity in Alzheimer's disease as measured by EEG coherence. J. Neurol. Neurosurg. Psychiatry 63 (1), 59-65.

Jeong, J., 2004. EEG dynamics in patients with Alzheimer's disease. Clin. Neurophysiol. 115, 1490-1505.

Kaminski, M.J., Blinowska, K.J., 1991. A new method of the description of the information flow in the structures. Biol. Cybern. 65, 203-210.

Kaminski, M.J., Blinowska, K.J., Szclenberger, W., 1997. Topographic analysis of coherence and propagation of EEG activity during sleep and wakefulness. Electroencephalogr. Clin. Neurophysiol. 102, 216227.

Kikuchi, M., Wada, Y., Koshino, Y., Nanbu, Y., Hashimoto, T., 2000. Effect of normal aging upon interhemispheric EEG coherence: analysis during rest and photic stimulation. Clin. Electroencephalogr. 31 (4), 170174

Kleiner, S., Bringmann, A., 1996. Nucleus basalis magnocellularis and pedunculopontine tegmental nucleus: control of the slow EEG waves in rats. Arch. Ital. Biol. 134, 153-167.

Klimesch, W., Doppelmayr, M., Pachinger, T., Russegger, H., 1997. Eventrelated desynchronization in the alpha band and the processing of semantic information. Brain Res. Cogn. Brain Res. 6 (2), 83-94.

Klimesch, W., Doppelmayr, M., Russegger, H., Pachinger, T., Schwaiger, J., 1998. Induced alpha band power changes in the human EEG and attention. Neurosci. Lett. 244 (2), 73-76.

Klimesch, W., 1999. EEG alpha and theta oscillations reflect cognitive and memory performance: a review and analysis. Brain Res. Rev. 29, 169-195.

Klimesch, W., 1996. Memory processes, brain oscillations and EEG synchronization. Int. J. Psychophysiol. 24 (1-2), 61-100.

Knott, V., Mohr, E., Mahoney, C., Ilivitsky, V., 2000. Electroencephalographic coherence in Alzheimer's disease: comparisons with a control group and population norms. J. Geriatr. Psychiat. Neurol. 13, 1-8.

Knyazeva, M.G., Innocenti, G.M., 2001. EEG coherence studies in the normal brain and after early-onset cortical pathologies. Brain Res. Brain Res. Rev. 36, 119-128.

Korzeniewska, A., Kasicki, S., Kaminski, M., Blinowska, K.J., 1997. Information flow between hippocampus and related structures during various types of rat's behavior. J. Neurosci. Meth. 73, 49-60.

Kus, R., Kaminski, M., Blinowska, K.J., 2004. Determination of EEG activity propagation: pair-wise versus multichannel estimate. IEEE Trans. Biomed. Eng. 51 (9), 1501-1510.

Lawton, M.P., Brodie, E.M., 1969. Assessment of older people: self maintaining and instrumental activity of daily living. J. Gerontol. 9, 179-186.

Leuchter, A.F., Newton, T.F., Cook, A.A., Walter, D.O., 1992. Changes in brain functional connectivity in Alzheimer-type and multi-infarct dementia. Brain 115, 1543-1561.

Locatelli, T., Cursi, M., Liberati, D., Francheschi, M., Comi, G., 1998. EEG coherence in Alzheimer's disease. Electroenceph. Clin. Neurophysiol. 106, 229-237.

McKahn, G., Drachman, D., Folstein, M., Katzman, R., Price, D., Stadlan, E.M., 1984. Clinical diagnosis of Alzheimer's disease: report of the NINCDS-ADRDA Work Group under the auspices of Department of Health and Human Services Task Force on Alzheimer's disease. Neurology 34, 939-944.

Mesulam, M., 2004. The cholinergic lesion of Alzheimer's disease: pivotal factor or side show? Learn. Mem. 11 (1), 9-43, Review.

Mima, T., Matsuoka, T., Hallett, M., 2000. Functional coupling of human right and left cortical motor areas demonstrated with partial coherence analysis. Neurosci. Lett. 287, 93-96.

Mima, T., Matsuoka, T., Hallett, M., 2001. Information flow from the sensorimotor cortex to muscle in humans. Clin. Neurophysiol. 112, 122-126. 
Montplaisir, J., Nielsen, T., Cote, J., et al., 1990. Interhemispheric EEG coherence before and after partial callosotomy. Clin. Electroencephalogr. $21,42-47$.

Moretti, D.V., Babiloni, F., Carducci, F., Cincotti, F., Remondini, E., Rossini, P.M., Salinari, S., Babiloni, C., 2003. Computerized processing of EEGEOG-EMG artifacts for multicentirc studies in EEG oscillations and event-related potentials. Int. J. Pshycophysiol. 47 (3), 199-216.

Nolte, G., Wheaton, O.B.L., Mari, Z., Vorbach, S., Hallett, M., 2004. Identifying true brain interaction from Eeg data using the imaginary part of coherency. Clin. Neurophysiol. 115, 2292-2307.

Pereda, E., Quian Quiroga, R., Bhattacharya, J., 2005. Nonlinear multivariate analysis of neurophysiological signals. Prog. Neurobiol. 77, 1-37.

Petersen, R.C., Smith, G.E., Ivnik, R.J., Tangalos, E.G., Schaid, D.J., Thibodeau, S.N., Kokmen, E., Waring, S.C., Kurland, L.T., 1995. Apolipoprotein E status as a predictor of the development of Alzheimer's disease in memory-impaired individuals. JAMA 273 (16), 1274-1278 (Erratum in: JAMA 274 (1995) (7) 538).

Petersen, R.C., Smith, G.E., Waring, S.C., Ivnik, R.J., Tangalos, E.G., Kokmen, E., 1999. Mild cognitive impairment: clinical characterization and outcome. Arch. Neurol. 56 (3), 303-308 (Erratum in: Arch. Neurol. 56 (1999) (6) 760).

Petersen, R.C., Doody, R., Kurz, A., Mohs, R.C., Morris, J.C., Rabins, P.V., Ritchie, K., Rossor, M., Thal, L., Winblad, B., 2001. Current concepts in mild cognitive impairment. Arch. Neurol. 58 (12), 1985-1992, Review.

Pfurtscheller, G., Lopez da Silva, F., 1999. Event-related EEG/MEG synchronization and desynchronization: basic principles. Clin. Neurophysiol. 110, 1842-1857.

Pijnenburg, Y.A., van der Made, Y., van Cappellen van Walsum, A.M., Knol, D.L., Scheltens, P., Stam, C.J., 2004. EEG synchronization likelihood in mild cognitive impairment and Alzheimer's disease during a working memory task. Clin. Neurophysiol. 115 (6), 1332-1339.

Pinkofsky, H.B., Struve, F.A., Meyer, M.A., et al., 1997. Decreased multiband posterior interhemispheric coherence with a lipoma on the corpus callosum: a case report of a possible association. Clin. Electroencephalogr. 28, 155-159.

Pogarell, O., Teipel, S.J., Juckel, G., Gootjes, L., Moller, T., Burger, K., Leinsinger, G., Moller, H.J., Hegerl, U., Hampel, H., 2005. Related articles links EEG coherence reflects regional corpus callosum area in Alzheimer's disease. J. Neurol. Neurosurg. Psychiatry 76 (1), 109-111.

Portet, F., Ousset, P.J., Visser, P.J., Frisoni, G.B., Nobili, F., Scheltens, P., Vellas, B., Touchon, J., 2006. Mild Cognitive Impairment in medical practice: critical review of the concept and new diagnostic procedure. Report of the MCI working group of the European Consortium on Alzheimer's disease (EADC). J. Neurol. Neurosurg. Psychiat. 77, 714-718.

Prichep, L.S., John, E.R., Ferris, S.H., Rausch, L., Fang, Z., Cancro, R., Torossian, C., Reisberg, B., 2006. Prediction of longitudinal cognitive decline in normal elderly with subjective complaints using electrophysiological imaging. Neurobiol. Aging 27, 471-481.

Ricceri, L., Minghetti, L., Moles, A., Popoli, P., Confaloni, A., De Simone, R., Piscopo, P., Scattoni, M.L., di Luca, M., Calamandrei, G., 2004.
Cognitive and neurological deficits induced by early and prolonged basal forebrain cholinergic hypofunction in rats. Exp. Neurol. 189 (1), $162-172$.

Rodriguez, E., George, N., Lachauz, J.P., Martinerie, J., Renault, B., Varela, F.J., 1999. Perception's shadow: long distance synchronization of human brain activity. Nature 397, 430-433.

Rosen, W.G., Terry, R.D., Fuld, P.A., Katzman, R., Peck, A., 1980. Pathological verification of ischemic score in differentiation of dementias. Ann. Neurol. 7 (5), 486-488.

Rossini, P.M., Desiato, M.T., Lavaroni, F., Caramia, M.D., 1991. Brain excitability and electroencephalographic activation: non-invasive evaluation in healthy humans via transcranial magnetic stimulation. Brain Res. 567 (1), 111-119.

Sarnthein, J., Petsche, H., Rappelsberger, P., Shaw, G.L., von Stein, A., 1998. Synchronization between prefrontal and posterior association cortex during human working memory. Proc. Natl. Acad. Sci. U.S.A. 95, 7092-7096.

Singer, W., 2001. Consciousness and the binding problem. Ann. N.Y. Acad. Sci. 929, 123-146.

Stam, C.J., van der Made, Y., Pijnenburg, Y.A.L., Scheltens, Ph., 2003. Synchronization of brain activity in mild cognitive impairment and early Alzheimer's disease. Acta. Neurol. Scand. 108 (2), 90-96.

Stam, C.J., van Dijk, B.W., 2002. Synchronization likelihood: an un-biased measure of generalized synchronization in multivariate data sets. Physica D 163, 236-251.

Steriade, M., Llinas, R.R., 1988. The functional states of the thalamus and the associated neuronal interplay. Physiol. Rev. 68 (3), 649-742.

Steriade, M., 2003. Neuronal Substrates of Sleep and Epilepsy. Cambridge University Press, Cambridge (UK), p. 522.

Sulimov, A., 1998. Human scalp-recorded EEG may be a result of activity of weakly-coupled subsystems. Neurosci. Lett. 250 (1), 72-74.

Teipel, S.J., Hampel, H., Pietrini, P., et al., 1999. Region-specific corpus callosum atrophy correlates with the regional pattern of cortical glucose metabolism in Alzheimer disease. Arch. Neurol. 56, 467-473.

Wada, Y., Nanbu, Y., Kikuchi, M., Koshino, Y., Hashimoto, T., Yamaguchi, N., 1998a. Abnormal functional connectivity in Alzheimer's disease: intrahemispheric EEG coherence during rest and photic stimulation. Eur. Arch. Psychiat. Clin. Neurosci. 248, 203-208.

Wada, Y., Nanbu, Y., Koshino, Y., Yamaguchi, N., Hashimoto, T., 1998b. Reduced interhemispheric EEG coherence in Alzheimer disease: analysis during rest and photic stimulation. Alzheimer Dis. Assoc. Disord. $12,175-181$.

Xiang, Z., Huguenard, J.R., Prince, D.A., 1998. Cholinergic switching within neocortical inhibitory networks. Science 281, 985-988.

Yamauchi, H., Fukuyama, H., Harada, K., et al., 1993. Callosal atrophy parallels decreased cortical oxygen metabolism and neuropsychological impairment in Alzheimer's disease. Arch. Neurol. 50, 10701074.

Yesavage, J.A., Brink, T.L., Rose, T.L., Lum, O., Huang, V., Adey, M., Leirer, V.O., 1982-1983. Development and validation of a geriatric depression screening scale: a preliminary report. J. Psychiatr. Res. 17 (1), 37-49. 